\title{
Escape dynamics in moderately damped Josephson junctions \\ (Review Article)
}

\author{
D. Massarotti ${ }^{1,2}$, L. Longobardi ${ }^{3}$, L. Galletti ${ }^{1,2}$, D. Stornaiuolo ${ }^{1,2}$, D. Montemurro ${ }^{4}$, \\ G. Pepe ${ }^{1,2}$, G. Rotoli $^{5}$, A. Barone $^{1,2}$, and F. Tafuri ${ }^{5,2,1}$ \\ ${ }^{l}$ Dip. Scienze Fisiche, Universitá di Napoli Federico II, Piazzale Tecchio 80, Napoli 80125, Italy \\ ${ }^{2}$ CNR-SPIN, UOS Napoli, Monte S. Angelo via Cinthia, Napoli 80126, Italy \\ ${ }^{3}$ Department of Physics and Astronomy, Dartmouth College, Hanover NH 03755, USA \\ ${ }^{4}$ NEST and Scuola Normale Superiore, Piazza San Silvestro 12, I-56127 Pisa, Italy \\ ${ }^{5}$ Dip. Ingegneria dell'Informazione, Seconda Universitá di Napoli, via Roma 29, Aversa (CE) 81031, Italy \\ E-mail: tafuri@na.infn.it
}

Received February 15, 2012

\begin{abstract}
The Josephson effect is a privileged access to the macroscopic quantum nature of superconductors. We review some ideas and experimental techniques on macroscopic quantum decay phenomena occurring in Josephson structures. The attention is mainly addressed to intermediate levels of dissipation which characterize a large majority of low critical current Josephson devices and are therefore an avoidable consequence of nanotechnology applied more and more to Josephson devices. Phase diffusion phenomena take over thermal activation in some temperature ranges also affecting the transition to macroscopic quantum tunneling, enriching the phase diagram mostly defined by the Josephson energy, the temperature and the level of dissipation.
\end{abstract}

PACS: 74.72.-h Cuprate superconductors;

74.50.+r Tunneling phenomena; Josephson effects;

74.40.-n Fluctuation phenomena.

Keywords: Josephson effect, SQUIDs, macroscopic quantum tunneling.

\section{Contents}

1. Introduction 336

2. A ball rolling down a washboard potential: from thermal activation to macroscopic quantum

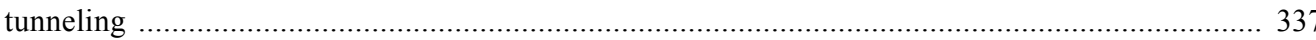

2.1. Formalism and the first measurements on switching current distributions .................................... 337

2.2. Josephson junctions as a part of a qubit ................................................................................. 339

3. Phase dynamics in "novel" types of junctions and the moderately damped regime ............................ 339

3.1. Macroscopic quantum phenomena in HTS grain boundary Josephson junctions ........................ 340

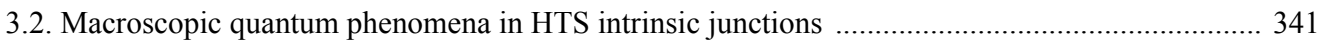

3.3. The moderately damped regime in low critical currents Josephson junctions ............................... 341

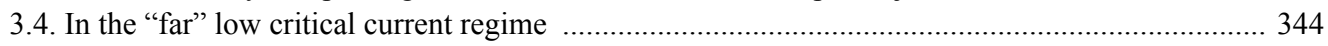

3.5. Switching current distribution measurements in "nanostructures" ................................................. 344

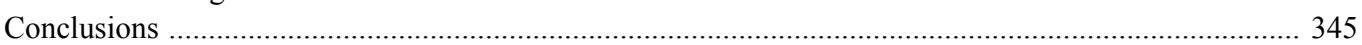

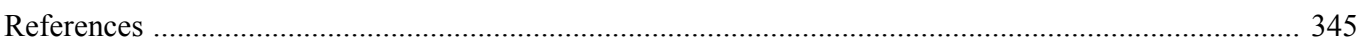

\section{Introduction}

The Josephson effect [1] and the physics of Josephson devices [2-4] have been in these last 50 years a continuous source of inspiration and progress in physics. We have been learning by investigating a Josephson device, how macro- scopic coherence propagates in heterostructures [2-5], how macroscopic and microscopic phases coherently combine at superconductor/normal metal $(\mathrm{S} / \mathrm{N})$ interfaces [6] of a S/N/S Josephson junction, how a Josephson coupling can unambiguously identify unconventional symmetry of the 
order parameter, as occurring in high critical temperature superconductors (HTS) [7]. The intrinsic and robust quantum nature of the Josephson junctions is also the basis of well established applications such as the superconducting quantum interference devices (SQUIDs) $[3,8]$ and of a prospective hybrid architecture for quantum information [9]. These are just a few examples chosen in a multitude of possibilities.

In this contribution we revise some of the main concepts and experimental procedures on which the studies on escape dynamics in Josephson devices are funded [10-12]. We look at escape dynamics aware that material science is not only offering a variety of novel interfaces and junctions, but also radically new solutions of synthesizing hybrid Josepshon devices taking advantage at the same time of the progress registered in using nanotechnologies in superconducting electronics. We are specifically interested in discussing escape dynamics at intermediate levels of dissipation (moderately damped regime), which is going to permeate more and more the nature of futuristic superconducting hybrid nanostructures.

This topic has been of high interest since the early eighties, with the first experimental evidence of quantum behavior in a Josephson tunnel junction displaying energy levels quantization and macroscopic quantum tunneling, which showed unequivocally that the difference in the phase of the Ginzburg-Landau [13] wavefunction in the two electrodes of a Josephson junction behaves as a quantum variable. This concept has more recently been extended to the implementation of superconductive quantum bits and in a whole series of beautifully designed experiments displaying a high degree of control of the energy level landscape.

\section{A ball rolling down a washboard potential: from thermal activation to macroscopic quantum tunneling}

\subsection{Formalism and the first measurements on switching current distributions}

Considerable insights into the nonlinear dynamics of a Josephson junction can be gained by realizing that the equation resulting from the resistively shunted junctions (RSJ) model [2-4]

$$
\left(\frac{\Phi_{0}}{2 \pi}\right)^{2} C \frac{\partial^{2} \phi}{\partial t^{2}}+\left(\frac{\Phi_{0}}{2 \pi}\right)^{2} \frac{1}{R} \frac{\partial \phi}{\partial t}+\frac{\partial}{\partial \phi} U=0
$$

where

$$
U=-\Phi_{0} /(2 \pi)\left(I_{c} \cos (\phi)+I \phi\right)
$$

with $\Phi_{0}$ the flux quantum and $I_{c}$ the critical current, describes the motion of a ball moving on the "tilted washboard" potential $U$ (see Figs. 1,a,b). The term involving the capacitance $C$ represents the mass of the particle, the $1 / R$ term represents the damping of the motion, and the average "tilt" of the washboard is propor-

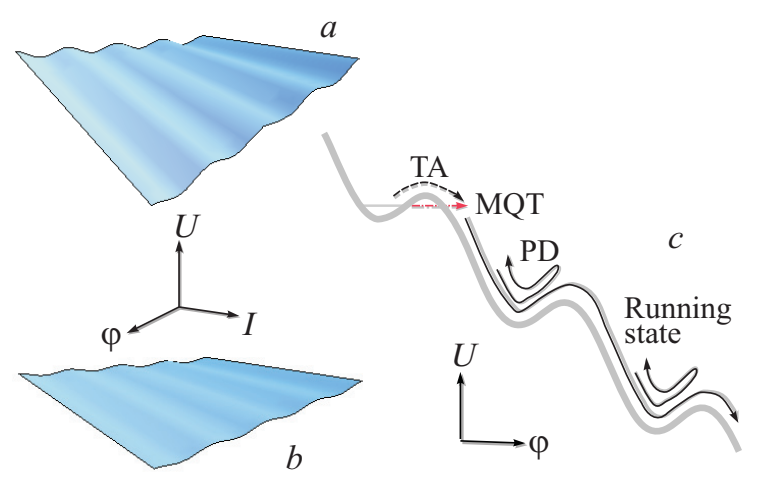

Fig. 1. (a) 3-dim view of wash-board potential in the RSJ model as a function of the phase and of the bias current. The current spans from 0 to $I_{c}$. In $(b) E_{J}$ is half of the value used in (a), and this favors phase diffusion regime (see text). (c) A 2-dim cut from $(b)$ for a fixed value of the current. TA stands for thermal activation (black dotted line), while MQT for macroscopic quantum tunneling (red dotted line). Once the particle/phase overcomes the barrier, it rolls in the running state. Retrapping processes may happen for intermediate levels of dissipation determining a phase diffusion $(\mathrm{PD})$ regime.

tional to $-I$. The strength of the friction can be also expressed through the junction quality factor $Q=\omega_{p} R C$, where $\omega_{p}=\left(2 e I_{c} / \hbar C\right)^{1 / 2}\left(1-l^{2}\right)^{1 / 4}$ is the plasma frequency being $\imath=I / I_{c}$.

When the bias current is ramped from $l=0$ to $l<1$, the junction is in the zero-voltage state in absence of thermal and quantum fluctuations and the particle is confined to one of the potential wells, where it oscillates back and forth at the frequency $\omega_{p}$. At finite temperature, the junction may switch into a finite voltage state for a bias current $l<1$. This corresponds to the particle escaping from the well either by a thermally activated (TA) process [14] or by tunneling through the barrier potential, known as macroscopic quantum tunneling (MQT) $[5,9,11,12]$ (see Fig. 1,c). This only occurs if the kinetic energy gained from running "down hill" in the tilted-washboard potential is not all dissipated, but enough remains to carry the representative point over the next "hill". The $Q$ quality factor measures the damping of the plasma oscillation by the effective shunt resistance $R$ of the junction and estimates the number of periods during which the oscillation energy will be dissipated. Once the phase point gets over a hill by fluctuations, it keeps running, provided that the damping is below some critical value. Following an event of escape the particle may travel down the potential for a few wells and then be retrapped in one of the following minima of the potential [15]. At low bias the process of escape and retrapping may occur multiple times generating extensive diffusion of the phase until an increase of the tilt of the potential, due to a change in the bias current, raises the velocity of the particle and the junction can switch to the running state [15-19]. 
In the pure thermal regime, the escape rate for weak to moderate damping $(Q>1)$ is determined by [14]

$$
\Gamma_{t}(I)=a_{t} \frac{\omega_{p}}{2 \pi} \exp \left(-\frac{\Delta U(I)}{k_{B} T}\right)
$$

where $\Delta U(I)=\left(E_{J} 4 \sqrt{2} / 3\right)(1-\imath)^{3 / 2}$ is the barrier height for $l$ close to one and $E_{J}=\hbar I_{c} / 2 e$ is the Josephson energy. The escape rate will be dominated by MQT at low enough temperature [5,20]: for $Q>1$ and $\iota$ close to one it is approximated by the expression for a cubic potential

$$
\Gamma_{q}(I)=a_{q} \frac{\omega_{p}}{2 \pi} \exp \left[-7.2 \frac{\Delta U(I)}{\hbar \omega_{p}}\left(1+\frac{0.87}{Q}\right)\right],
$$

where $a_{q}=\left(864 \pi \Delta U / \hbar \omega_{p}\right)^{1 / 2}$.

The MQT rate is affected by dissipation, the irreversible energy transfer between the system and the environment, because of the damping dependent factor [5,20,21].

It is convenient to express the thermal and the quantum escape through an escape temperature $T_{\text {esc }}$ defined as

$$
\Gamma_{t, q}(I)=\frac{\omega_{p}}{2 \pi} \exp \left[-\frac{\Delta U(I)}{k_{B} T_{\mathrm{esc}}}\right]
$$

This is made possible because in both the classical and quantum regimes $T_{\text {esc }}$ is very nearly independent of the bias current. The crossover temperature $T_{\mathrm{cr}}$ between the thermal and quantum regimes is given by $T_{\text {cr }}=\left(\hbar \omega_{p} / 2 \pi k_{B}\right)\left\{\left(1+1 / 4 Q^{2}\right)^{1 / 2}-1 / 2 Q\right\}$.

An analytical expression for the retrapping rate from the resistive to the superconducting state is known only for strongly underdamped JJs:

$$
\Gamma_{R}=\omega_{p 0} \frac{I-I_{R}}{I_{c}} \sqrt{\frac{E_{J}}{2 \pi k_{B} T}} \exp \left[-\frac{E_{J} Q_{0}^{2}}{2 k_{B} T}\left(\frac{\left(I-I_{R}\right)^{2}}{I_{c}^{2}}\right)\right],
$$

where $I_{R}$ is the fluctuation-free retrapping current, $\omega_{p 0}$ and $Q_{0}$ are the plasma frequency and quality factor for $I=0$, respectively.

The behavior of the phase difference $\varphi$ is deduced from measurements of the escape rate $\Gamma$ of the junctions from its zero-voltage state. To determine the escape rate $10^{4}-10^{5}$ events are typically collected for each set of parameters. The resulting distribution of the switching probability $P(I)$ is used to compute the escape rate out of the zero-voltage state as a function of the bias current $I$ following Fulton and Dunkleberger [10]:

$$
\Gamma(I)=\frac{1}{\Delta I} \frac{d I}{d t} \ln \left(\frac{\sum_{i \geq I} P(I)}{\sum_{i \geq I+\Delta I} P(I)}\right),
$$

where $d I / d t$ is the current ramp rate and $\Delta I$ is the channel width of the analog-to-digital converter.

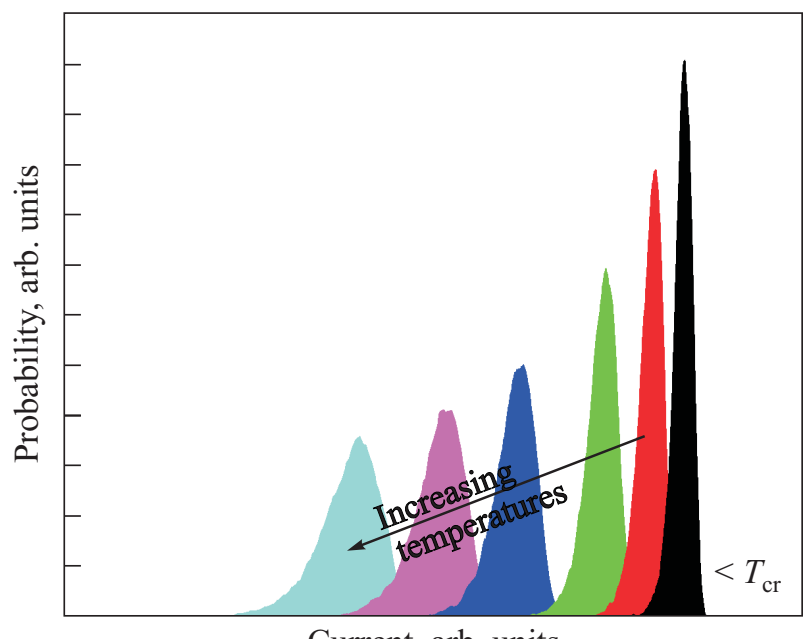

Current, arb. units

Fig. 2. Schematic representation of SCD measurements from the thermal regime to macroscopic quantum tunneling.

The very first experiments on MQT in a Josephson junction were carried out by Voss and Webb [22] and by Jackel et al. [23], while related experiments on a junction inserted in a superconducting loop were realized by de Bruyn Ouboter et al. [24], Prance et al. [25] and Dmitrenko et al. [26]. Switching current distribution histograms present a characteristic behavior reported schematically in Fig. 2. By lowering the temperature $T$ the histograms move to higher currents and their width $\sigma$ scales with the temperature down to $T_{\mathrm{cr}}$. For $T<T_{\mathrm{cr}}, \sigma$ is independent of $T$. The temperature dependence of the effect of damping on the tunneling has been addressed by later experiments [27,28]. Devoret, Martinis and Clarke [11,12] have established a detailed conceptual and experimental protocol to follow to prove the macroscopic quantum nature of tunneling and its crossover to the thermal regime, used in most of later experiments. It has been clearly addressed the problem of the complex impedance presented to the junction at microwave frequencies by the wires directly connected to it or by any circuit in its vicinity, and classical phenomena have been used to measure all relevant parameters of the junction in situ [11,12]. The relevant parameters of the junction (critical current and shunting admittance) were determined in situ in the thermal regime from the dependence of $\Gamma$ on bias current and from resonant activation in the presence of microwaves. The shunting capacitance dominated the self-capacitance of the junction, while the bias circuitry determined the shunting conductance, respectively. The magnetic field has been used as a knob to tune the crossover temperature by changing the critical current and therefore the plasma frequency.

In a further series of experiments, the existence of quantized energy levels in the potential well of the junction was demonstrated spectroscopically [11,12]. The escape rate from the zero-voltage state was increased when the microwave frequency $\Omega$ corresponded to the energy difference between two adjacent energy levels. A crucial 
point is that the anharmonic nature of the well, which results from the nonlinear inductance of Josephson junctions, causes the energy spacing to decrease as the quantum number progressively increases, so each transition has a distinct frequency. The transition may involve more than one photon at once, thus called multiphoton transition, which has been observed experimentally [29].

This set of experiments clearly proved that $\varphi$ is a quantum variable. Although this system contains a large number of atomic constituents, it is atomlike in the sense that it has a single degree of freedom behaving quantum mechanically. Thermal energy must be sufficiently low to avoid incoherent mixing of eigenstates, and the macroscopic degree of freedom must be sufficiently decoupled from other degrees of freedom for the lifetime of the quantum states to be long on the characteristic time scale of the system [5].

\subsection{Josephson junctions as a part of a qubit}

Superconducting qubits are solid state electrical circuits that exploits the numerous advantages of using superconductors, as well as the macroscopic quantum behavior of devices based on Josephson tunnel junctions, to engineer quantum states suitable for quantum computing. Superconductors are particularly fit to be used for quantum integrated circuits since they allow for design and fabrication using techniques that can be borrowed from conventional integrated circuits, and they have intrinsically low dissipation and low noise due to the fact that the experiments are often conducted at millikelvin temperatures. Furthermore, as explained in the previous section, the Josephson potential is highly anharmonic, which allows to selectively excite the states used as basis of the qubit, and to avoid contributions from other energy levels.

Macroscopic quantum coherence, i.e., the superposition of two macroscopically distinctive quantum states $\left|\Psi_{1}\right\rangle$ and $\left|\Psi_{2}\right\rangle$ in the form $|\Psi\rangle=\alpha\left|\Psi_{1}\right\rangle+\beta\left|\Psi_{2}\right\rangle$ [30] is a fundamental component of quantum computation, and was first demonstrated by Nakamura et al. in 1997 with the first experiment on a charge qubit [31], showing spectroscopically the superposition of the Cooper-pair states $|n\rangle$ and $|n+1\rangle$, where the integer $n$ is the quantum number specifying the number of Cooper pairs. Demonstrations of the superposition of states in a flux qubit by the Stony Brook [32] and Delft [33] groups followed. A flux qubit consists of a superconducting loop interrupted by one [32] or three [33] Josephson junctions. The two quantum states consist of supercurrent flowing in an anticlockwise or clockwise direction or, equivalently, flux pointing up and flux pointing down, respectively. The Saclay group [34] realized in 2002 a qubit, named "quantronium", in which two small junctions are connected by a superconducting island, involving the superposition of the Cooper-pair states $|n\rangle$ and $|n+1\rangle$. In the phase qubit realized by Martinis et al. in 2002 [35], the relevant quantum states are the ground state and the first excited state, and the final device is basically the same used earlier to observe quantized energy levels [11,12].

These first experiments also classify the main three different types of qubit, i.e., charge, flux and phase, and have opened the way to a vast series of studies [9]. Measurements in the time domain for instance followed in order to determine the dynamical behavior of a qubit [36-38]. These experiments aimed to measure Rabi oscillations, spin echoes and Ramsey fringes require manipulating the state of the qubit by using appropriate microwave pulses, which are also the operative tools to implement single-qubit gates for quantum computing. Macroscopic resonant tunneling (MRT) when energy levels in each well are aligned, turns out to be an "accessible" reliable tool to perform on a routine basis the task to extract all devices quantum parameters, flux noise, and for a diagnostics of fabrication processes and materials $[39,40]$. This represents another example of how "quantum measurements" become more and more accessible, and a tool to drive material science choices on the nature and the performances of the junctions.

Another big force in driving research in the superconducting quantum measurements/qubit field has been to engineer systems in order to make them as less as possible sensitive to decoherence. This work has led to remarkable increases in decoherence times compared with those of early devices [9]. The development of more advanced charge qubits such as the transmon [41] and the quantronium [34] are examples of improved charge qubits, where attention is paid to protect the devices from low-frequency noise and from electrons moving among defects. Future implementations of quantum information processing will try to promote hybrid systems. The idea is to combine microscopic systems such as atoms or spins which are naturally well decoupled from their environment and can reach extremely long coherence times, with more macroscopic objects such as superconducting circuits which are strongly coupled to electromagnetic fields, making them easy to entangle although with shorter coherence times [42].

\section{Phase dynamics in "novel" types of junctions and the moderately damped regime}

The idea that performances of qubits could be improved and optimized by a suitable design of the ensemble qubit/cavity (circuit) was accompanied by the awareness that some limits on coherence are imposed by intrinsic dissipation due to the "chemistry" of the junctions, of the barrier interfaces and of the materials composing them. Progress in engineering new materials into junctions and in understanding and more and more controlling the physics of interfaces may offer novel solutions for junctions of superior quality and complementary functionalities, and therefore may lead in the long run to improve specific qubit performances. In other words, material science contributes to develop solutions for hybrid systems for quantum computation. 
In this section we focus on how experiments on the junction macroscopic quantum behavior have been extended to novel types of structures and materials. Switching current distribution (SCD) measurements in these last years have turned to be standard tools to investigate phase dynamics in unconventional and hybrid systems and nanostructures. High critical temperature superconductors (HTS) are an example of unconventional systems, because of the $d$-wave order parameter symmetry (OPS) and of the presence of low-energy quasiparticles [7], which are expected to induce high level of dissipation and as a consequence to spoil macroscopic quantum coherence.

For low critical temperature superconductors, once the barrier thickness and the critical current density $\left(J_{c}\right)$ have been fixed, a reduction in its size unavoidably leads to a lowering of the critical current and determines a quite different phase dynamics "re-normalized" to the new scaling energy. Lower critical currents $I_{c}$ result in lower Josephson energies $E_{J}$, and higher levels of dissipation are expected. The range of the energy dynamical parameters is significantly enlarged, and it is technologically easier to reproducibly realize nontrivial configurations. The pioneering studies of Kautz and Martinis [15] and Iansiti et al. [43] on small junctions where $E_{J}$ could be significantly lowered, can be now supported and developed by different types of junctions of quite different sizes. These devices are characterized by intermediate levels of dissipation (moderately damped regime) and by phase diffusion phenomena. The low $J_{c}$ limit seems to be characteristic also of all futuristic nanohybrids devices incorporating nanowires, and the moderately damped regime is intrinsically more common than it could be expected.

In summary we will refer as novel types of junctions, those composed of novel materials, or devices scaled to the nanosize or based on novel design concepts, as intrinsic junctions in HTS or junctions using nanowires as barriers.

\subsection{Macroscopic quantum phenomena in HTS grain boundary Josephson junctions}

The first examples of unconventional systems are given by HTS devices [44], biepitaxial grain boundary (GB) YBCO Josephson junctions $[45,46]$ and a variety of intrinsic junctions, built on high-quality single crystals $[47,48]$.

HTS may be an interesting reference system for novel ideas on key issues on coherence and dissipation in solid state systems because of their unusual properties $[7,49]$. Low-energy quasiparticles have represented since the very beginning a strong argument against the occurrence of macroscopic quantum effects in these materials. Quantum tunnelling of the phase leads to fluctuating voltage across the junctions which excites the low-energy quasiparticles specific for $d$-wave junctions, causing decoherence. Contributions to dissipation due to different transport processes, such as channels due to nodal quasiparticles, midgap states, or their combination, have been identified and distinguished [50].
The search of macroscopic quantum effects become feasible once high-quality HTS Josephson junctions [44] with significant hysteresis in the current-voltage (I-V) characteristics were available. We can distinguish two classes of experiments, which are based on two different complementary types of junctions: 1) MQT and energy level quantization (ELQ) $[45,46]$ on off-axis YBCO grain boundary biepitaxial JJs, where the experiment has been designed to study $d$-wave effects with a lobe of the former electrode facing the node of the latter; 2) MQT and ELQ on intrinsic junctions on single crystals of different materials $[47,48]$, where $d$-wave effects are expected to play a minor role [50]. The experiments using GBs are more complicated because of the complexity of these junctions, but are very complete and allow to address relevant issues on the effects of a $d$-wave OPS on dissipation and coherence. Only GBs junctions can be more easily integrated into circuits.

In HTS Josephson junctions, when a lobe of the OPS on one side of the junction meets a lobe on the other side, a larger critical current density $\left(J_{c}\right)$ is measured, differently from the case of a lobe facing a node [7,44,51]. If we produce $d$-wave junctions with different interface orientations, as for instance made possible by the biepitaxial technique, a wide range of different OPS configurations are realized, representing an additional knob to tune phase dynamics. The interest for this "intrinsically encoded phase dynamics" cannot be disjointed from the general problem of understanding dissipation in systems with very low energy and potentially highly dissipative quasiparticles, as occurring in $d$-wave systems. Since the frequency dependent quality factor $Q(\omega)=\omega_{p} R(\omega) C$ [15] is a measure of dissipation in the JJ, while the critical current $I_{c}$ is an easily accessible parameter, the dissipative essence strongly depends on the value of the effective frequency dependent resistance $R(\omega)$ and of shunting capacitance $C$, which in turn depend on several interplaying effects, such as circuit impedance, subgap resistance and stray capacitance. Since these parameters are not easily accessible, a reliable way able to estimate the frequency dependent quality factor $Q(\omega)$ is of great interest.

The GB biepitaxial junctions $[52,53]$ used in $[45,46]$ had reproducible hysteretic behavior up to $90 \%$. A specific feature of these structures is the use of a (110)-oriented $\mathrm{CeO}_{2}$ buffer layer, deposited on (110) $\mathrm{SrTiO}_{3}$ substrates. YBCO grows along the [001] direction on the $\mathrm{CeO}_{2}$ seed layer, while it grows along the [103]/[013] direction on $\mathrm{SrTiO}_{3}$ substrates $[53,54]$. The presence of the $\mathrm{CeO}_{2}$ produces an additional $45^{\circ}$ in-plane rotation of the YBCO axes with respect to the in-plane directions of the substrate. Atomically flat interfaces can be achieved in appropriate conditions [52]. This configuration produces a $45^{\circ}$ misorientation between the two electrodes to enhance $d$-wave order parameter effects, by varying the interface orientation. 
The junction in the tilt configuration (angle $\theta=0^{\circ}$ ) turns out to be the most interesting case for the MQT and ELQ experiments. This lobe to node configuration maximizes $d$-wave induced effects and allows to explore the effects of low-energy quasiparticles. The switching current probability distributions as a function of temperature for the biepitaxial JJ substantially follows what commonly measured on LTS JJs, with a saturation of the measured $\sigma$ below $50 \mathrm{mK}$ [45], which corresponds to the crossover temperature from the thermal to the MQT regime and is consistent with the predicted values. The change of $T_{\mathrm{cr}}$ through an external magnetic field is an important confirmation of the occurrence of MQT. Values of $R \simeq 100 \Omega$ and $C_{J} \sim 0.22 \mathrm{pF}$ can be obtained from the measurements with a plasma frequency $\omega_{p} / 2 \pi \simeq 2.6 \mathrm{GHz}$ and a quality factor of the order of $10[45,46,55]$. $C$-axis tilt is mostly responsible for low-barrier transparency and leads to the presence of a significant kinetic inductance in the modeling of YBCO JJ. In these junctions the presence of a kinetic inductance and a stray capacitance determine the main difference in the washboard potential making the system behavior depending on two degrees of freedom $[46,55]$. The YBCO JJ is coupled to this LC-circuit and the potential become two-dimensional (2D).

In summary macroscopic quantum phenomena have observed also in HTS in extremely unfavorable conditions of lobe-node configuration and therefore nominally in presence of low-energy quasiparticles $[45,46]$.

\subsection{Macroscopic quantum phenomena in HTS intrinsic junctions}

Experiments on $\mathrm{Bi}_{2} \mathrm{Sr}_{2} 2 \mathrm{CaCu}_{2} \mathrm{O}_{8}$ intrinsic Josephson junctions (IJJ) $[47,48]$ have been aimed to increase the crossover temperature $\left(T_{\mathrm{cr}}\right)$ and to clarify the nature of IJJs, rather than raising novel themes of coherence in $d$-wave systems. In these junctions the nodes of the $d$-wave order parameter are not expected to affect significantly MQT. Josephson coupling between $\mathrm{CuO}_{2}$ double layers has been proved, and most of the materials behaved like stacks of S-I-S JJs with effective barriers of the order of the separation of the $\mathrm{CuO}_{2}$ double layers $(1.5 \mathrm{~nm})\left(J_{c}\right.$ typically $\left.10^{3} \mathrm{~A} / \mathrm{cm}^{2}\right)[56,57]$. IJJs have a much higher Josephson coupling energy than GB junctions, the I-V curves exhibited large hysteresis and multiple branches, indicative of a series connection of highly capacitive junctions. Practical realizations of IJJ have been designed in order to nominally avoid heating effects [18,57]. However, at high-voltages caution is required when extracting information because of possible unavoidable heating problems. $T_{\mathrm{cr}}$ has been reported to be about $800 \mathrm{mK}$, remarkably higher than those usually found in LTS systems. By using microwave spectroscopy, the unique uniform array structure of intrinsic Josephson junction stacks have been considered responsible for a remarkable enhancement of the tunneling rate [48]. This enhancement adds a factor of approximately $N^{2}$ to the quantum escape rate of a single Josephson junction, also resulting in a significant increase of $T_{\mathrm{cr}}$, where $N$ is the number of the junctions in the stack. This effect can be caused by large quantum fluctuations due to interactions among the $N$ junctions [48]. Intrinsic junctions have been also studied in the moderately damped regime as discussed in the next section.

\subsection{The moderately damped regime in low critical currents Josephson junctions}

The possibility to reproducibly achieve low critical currents in submicron junctions promoted studies on moderately damped junctions $(1<Q<10)$. The same regime can be controllably induced in larger junctions in case of low critical current densities, or with lower reproducibility in junctions with larger intrinsic dissipation levels, as occurring in HTS systems.

As it can be seen in Fig. 1,c, the diffusive regime is characterized by the onset of multiple retrapping processes in subsequent potential wells. As roughly sketched in Fig. 1,b and by the Eq. (6), a decrease of the Josephson energy $E_{J}$ and of the quality factor $Q$ enhances the retrapping probability $\Gamma_{R}$, causing multiple retrapping phenomena in the switching dynamics.

As opposed to the strongly underdamped Josephson junctions case, well described by the analysis of Fulton and Dunkleberger [10] in the thermal region, overdamped junctions show nonhysteretic behavior, with a finite voltage on the supercurrent branch of the I-V characteristic, associated with thermally activated phase diffusion, and thermal fluctuations leading to very much smaller variations in the switching behavior. If the damping around the junction plasma frequency is sufficiently high, at low bias the phase particle will always retrap in a local minima after escape and a finite resistance phase-diffusion branch appears on the I-V curve of the junction (the appearance of a small voltage, prior to switching to a voltage on the order of twice the superconducting gap $\Delta$ ). Phase diffusion (PD) in junctions with hysteretic $\mathrm{I}-\mathrm{V}$ characteristics has been discussed in literature $[15,58]$ and has been associated with frequency-dependent damping, so that junctions are underdamped at high frequencies but are in the overdamped limit at low frequencies, respectively.

Phase diffusion process has reappeared in a different regime in magnetometers with much larger and unshunted junctions used for qubits readout [17]. The retrapping process is affected by the frequency dependent impedance of the environment of the dc SQUID. For $Q>1$ with relatively small $I_{c}$, such that $E_{J} \sim k_{B} T>E_{c}$, it has been shown that a regime exists where escape does not lead to a finite-voltage state, but rather to underdamped phase diffusion [16]. In addition to the usual crossover between MQT and TA behavior, the transition from TA behavior to underdamped phase diffusion has been also observed, resulting in a more diversified $\left(k_{B} T, E_{J}\right)$ phase diagram [16]. 
The transition from TA to underdamped phase diffusion regime is marked by the collapse of the width of the switching distributions and the turn over temperature $T^{*}$ is defined as the temperature at which the width $\sigma$ of the switching current distribution reaches the maximum value (see below). The change in the sign of the derivative of the second moment of the distribution and a modification of the shape of the distributions at temperatures around $T^{*}$ turn to be distinctive signatures of the PD regime. The phase diagram in [16] defines the relation between $E_{J}$ and the turn over temperature $T^{*}$ and indicates how the transition from TA to underdamped phase diffusion can be tuned by the critical current of the JJ.

In [18] a systematic analysis has been carried out on different types junctions, all with low values of the critical current: low ohmic $\mathrm{Nb}-\mathrm{Pt}-\mathrm{Nb}(\mathrm{S}-\mathrm{N}-\mathrm{S})$ junctions, $\mathrm{Nb}-\mathrm{CuNi}-\mathrm{Nb}(\mathrm{S}-\mathrm{F}-\mathrm{S})$ junctions with a diluted ferromagnetic alloys, $\mathrm{Nb}-\mathrm{InAs}-\mathrm{Nb}$ (S-2D electron gas-S) structures and $\mathrm{BiSrCaCuO}$ (2212) IJJ. The damping parameter of the junctions has been tuned in different manners by changing temperature, magnetic field, and gate voltage and introducing a ferromagnetic layer or in situ capacitive shunting. The phenomenon of an unexpected collapse of switching current fluctuations with increasing $T$ is explained by the interplay of two counteracting consequences of thermal fluctuations [18]. On one hand, thermal fluctuations assist in premature switching into the resistive state and, on the other hand, help in retrapping back to the superconducting state [18]. In other words, temperature does not only provide energy for excitation of a system from equilibrium state but also enhances the rate of relaxation back to the equilibrium. Multiple-retrapping processes in a hysteretic $\mathrm{BiSrCaCuO}$ (2212) IJJ with a high tunneling resistance have been reported to govern the switching from a resistive state in the phase-diffusion regime into the quasiparticle tunneling state [59]. The frequency-dependent junction quality factor, representing the energy dissipation in a phase-diffusion regime, determines the observed temperature dependence of the switching current distribution and the switching rate. Phase dynamics has been investigated also in underdamped ferromagnetic JJ by measuring the switching probability in both the stationary and nonstationary regimes $[60,61]$. The junction is $\mathrm{Nb}-\mathrm{Al}_{2} \mathrm{O}_{3}-\mathrm{PdNi}$ $(10 \% \mathrm{Ni})-\mathrm{Nb}$. Incomplete relaxation leads to dynamical phase bifurcation. Bifurcation manifests itself as a premature switching, resulting in a bimodal switching distribution [60]. Escape rate measurements at temperatures $T$ down to $20 \mathrm{mK}$ show that the width of the switching current histogram decreases with temperature and saturates below $T=150 \mathrm{mK}$ in $\mathrm{Nb} / \mathrm{Al}_{2} \mathrm{O}_{3} / \mathrm{Cu}_{40} \% \mathrm{Ni}_{60} \% / \mathrm{Nb}$ (superconductor-insulator-ferromagnet-superconductor) heterostructures [61].

Distinctive fingerprints of phase diffusion can be found in the temperature dependence of switching current distribution (SCD) histograms and of their width $\sigma$. An exam- ple is shown in Fig. 3 for $\mathrm{NbN} / \mathrm{MgO} / \mathrm{NbN}$ JJs $[62,63]$. SCD curves are reported for zero $(H=0)$ and for finite $(H=6.09 \mathrm{G})$ magnetic field in Figs. 3, $a$ and 3,b, respectively. The temperature dependence of $\sigma$ is given in the upper frame of Fig. 4. We can clearly distinguish at lower temperatures the transition from MQT to TA. When increasing the temperature, histograms tend to become more symmetric and shrink rather than broaden with a consequent increase of their maximum amplitude $[62,63]$. This translates in a characteristic dependence of $\sigma$, i.e, appearance of an anticorrelation between the temperature and the width of the switching distributions [16-19,62,63]. After the MQT saturation at the lowest temperatures (in this case about $100 \mathrm{mK}), \sigma$ follows the expected $T^{2 / 3}$ dependence, deviations are evident in proximity and above the crossover temperature $T^{*}$ where the temperature derivative of $\sigma(T)$ becomes negative. The $\mathrm{MgO}$ barrier is $1 \mathrm{~nm}$ thick in this case, providing $J_{c}$ of about $3 \mathrm{~A} / \mathrm{cm}^{2}$. The total current $I_{c}$ is about $2 \mu \mathrm{A}$ and falls under the criteria of the moderately damped regime. The experimental data are well
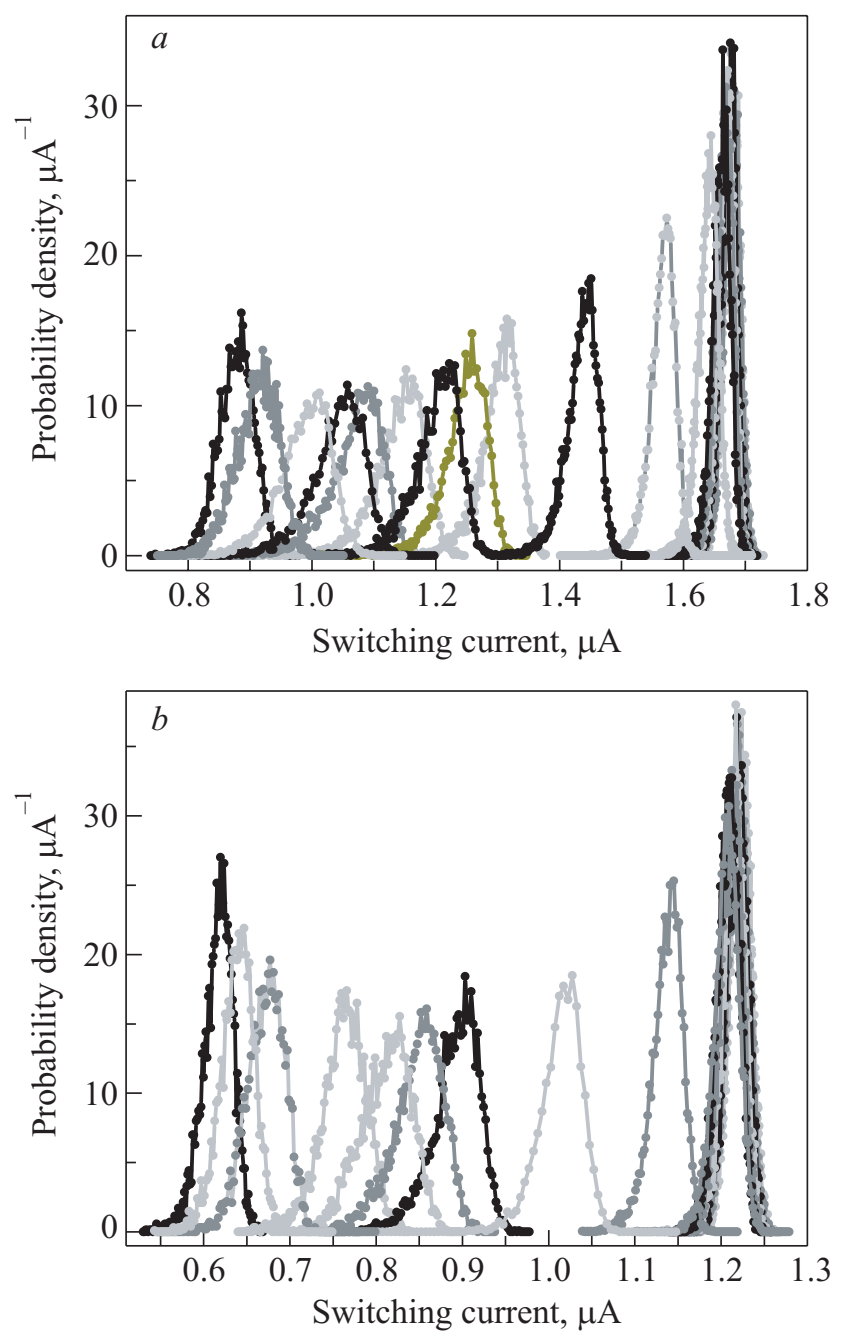

Fig. 3. Switching current probability distribution at $H=0 \quad(a)$ and $H=6.09 \mathrm{G}(b)$ of a $\mathrm{NbN} / \mathrm{MgO} / \mathrm{NbN} \mathrm{JJ}[62,63]$. 
reproduced by the expected values, as shown in the lower frame of Fig. 4, calculated on the basis of the physical arguments of phase diffusion [63].

The simulations are based on the recent work on phase diffusion by Fenton and Warburton (FW) [19]. The phase difference $\varphi(t)$ is a solution of the following Langevin differential equation:

$$
\varphi_{t t}+\varphi_{t} / Q+\imath+\imath_{N}=0
$$

Times $t$ are normalized to $1 / \omega_{p} ; \quad l$ is the bias current normalized to critical current $I_{c 0}$ and $l_{N}$ is a Gaussian correlated thermal noise current, i.e.:

$$
\left\langle\iota_{N}(t), \iota_{N}\left(t^{\prime}\right)\right\rangle=\sqrt{2 \pi k_{B} T / Q I_{c 0} \Phi_{0}} \delta\left(t-t^{\prime}\right) .
$$

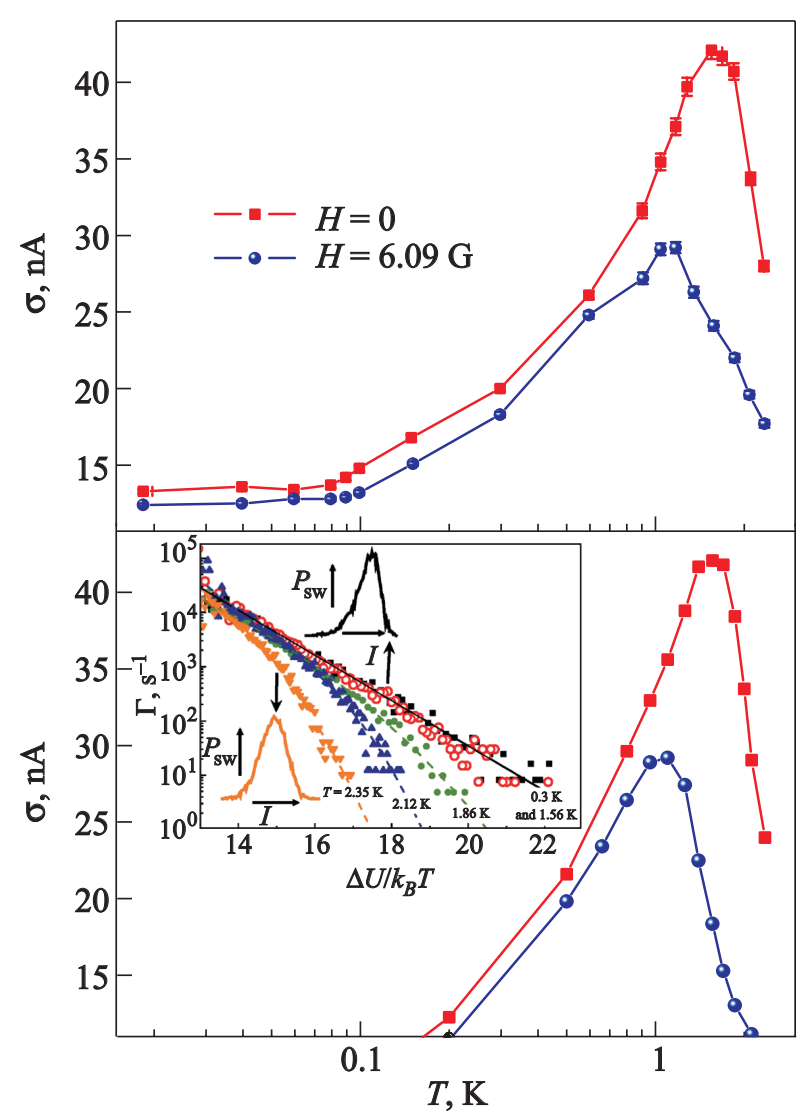

Fig. 4. Top frame: temperature dependence of the standard deviation $\sigma$ of the switching distributions for $H=0 \mathrm{G}$ (squares) and $H=6.09 \mathrm{G}$ (circles) shown in Fig. 3. Bottom frame: a numerical simulation of the data. In the inset escape rates (symbols) as a function of the barrier height at zero magnetic field for temperatures near $T^{*}$ are shown, together with numerical simulations (dashed lines).
Stochastic dynamics is simulated by integrating the above Langevin equation by a Bulirsh-Stoer integrator using as noise generator the cernlib routine RANLUX [64]. The magnetic field works as a knob to tune $T^{*}$ and provides an additional validity test for the estimate of $Q=2.7 \pm 0.1[62]$.

Phase diffusion also appears in the escape rates $\Gamma$, shown in the inset of Fig. 4 as a function of the ratio between the barrier height $\Delta U$ and the thermal energy. The escape rates are calculated from the switching distributions using Eq. (7). In the thermal activation regime the distributions are asymmetric and skewed to the left, and $\Gamma$ values all fall onto the same line, as it is the case for the reported data from $T=0.3 \mathrm{~K}$ to $1.56 \mathrm{~K}$. Retrapping processes cause a progressive symmetrization of the switching distribution, as it can be seen in Figs. 3, $a$ and 3,b, and a bending in the $\Gamma$ vs $\Delta U / k_{B} T$, as shown in the inset of Fig. $4[62,63]$.

The symmetrization of the switching distribution due to the interplay between escape and retrapping events can be clearly observed by plotting, as a function of temperature, the skewness of the distributions $\gamma$, i.e., the ratio $m_{3} / \sigma^{3}$ where $m_{3}$ is the third central moment of the distribution. For the lowest temperatures we obtain $\gamma=-1$, which is consistent with the case of switching current distributions in the quantum or thermal regime. As the temperature increases the distributions become more and more symmetric as $\gamma$ tends to zero $[62,63]$. It should be noted that for these data the temperature $T^{*}$ at which the derivative of $\sigma(T)$ changes sign is equal to $(1.62 \pm 0.3) \mathrm{K}$ and that the skewness starts increasing already at about $1.2 \mathrm{~K}$, which is a clear indication that the onset of retrapping phenomena occurs well below $T^{*}$ [19].

The very good fitting of experimental curves $[62,63]$ using the FW approach [19] confirms the occurrence of a multiple-retrapping regime with a large number of escapes of duration of $\Gamma_{R}^{-1}$, and in particular the relevance of the dependence of retrapping probability on time. The experimental results are well described by a numerical model involving a frequency independent damping which demonstrates an efficient way to estimate the dissipation in moderately damped JJs.

A nonexhaustive list of Josephson devices that have displayed a similar PD regime is reported in Table 1 along with the most relevant device parameters. Independently of the physical size of the device, all the junctions exhibiting phase diffusion over a large range of materials and geometry have a low critical current, $1<Q<6$ and $12<\Delta U(\bar{I}) / k_{B} T^{*}<18$, which are therefore relevant parameters signaling the insurgence of multiple escape and retrapping in a washboard potential. 
Table 1. Comparison of device parameters

\begin{tabular}{|c|c|c|c|c|c|}
\hline Author & Device structure & $\begin{array}{l}R, \\
\Omega\end{array}$ & $\begin{array}{c}I_{c 0}, \\
\mu \mathrm{A}\end{array}$ & $\begin{array}{l}C, \\
\mathrm{fF}\end{array}$ & $\begin{array}{c}Q \\
(I=0)\end{array}$ \\
\hline $\begin{array}{c}\text { Longobardi } \\
\text { et al. [63] }\end{array}$ & $\mathrm{NbN} / \mathrm{MgO} / \mathrm{NbN} \mathrm{JJ}$ & 65 & 1.91 & 300 & 2.7 \\
\hline $\begin{array}{l}\text { Kivioja } \\
\text { et al. [16] }\end{array}$ & $\mathrm{Al} / \mathrm{AlO}_{x} / \mathrm{Al} \mathrm{dc}$ SQUID & 500 & 0.2 & 100 & 3.9 \\
\hline $\begin{array}{l}\text { Kivioja } \\
\text { et al. [16] }\end{array}$ & $\mathrm{Al} / \mathrm{AlO}_{x} / \mathrm{Al} \mathrm{JJ}$ & 230 & 0.63 & 130 & 3.6 \\
\hline $\begin{array}{l}\text { Männik } \\
\text { et al. [17] }\end{array}$ & $\mathrm{Nb} / \mathrm{AlO}_{x} / \mathrm{Nb}$ dc SQUID & 70 & 4.25 & 90 & 2.4 \\
\hline $\begin{array}{c}\text { Männik } \\
\text { et al. [17] }\end{array}$ & $\mathrm{Nb} / \mathrm{AlO}_{x} / \mathrm{Nb}$ dc SQUID & 70 & 2.9 & 260 & 3.3 \\
\hline $\begin{array}{c}\text { Bae et al. } \\
{[59]^{*}}\end{array}$ & Bi-2212 intrinsic $\mathrm{JJ}$ & 62 & 1.26 & 330 & 2.2 \\
\hline $\begin{array}{c}\text { Yu et al. } \\
{[64]}\end{array}$ & $\mathrm{Nb} / \mathrm{AlO}_{x} / \mathrm{Nb} \mathrm{JJ}$ & 1800 & 0.122 & 20 & 4.8 \\
\hline $\begin{array}{c}\text { Yu et al. } \\
{[64]}\end{array}$ & $\mathrm{Nb} / \mathrm{AlO}_{x} / \mathrm{Nb} \mathrm{JJ}$ & 315 & 0.48 & 77 & 3.3 \\
\hline $\begin{array}{l}\text { Krasnov } \\
\text { et al. [18] }\end{array}$ & S-2DEG-S & 10 & 37 & 200 & 1.6 \\
\hline $\begin{array}{l}\text { Krasnov } \\
\text { et al. [18] }\end{array}$ & Bi-2212 intrinsic JJ & 40 & $80 * *$ & 685 & 5.6 \\
\hline
\end{tabular}

* In this paper the authors estimated the fit parameters to be temperature dependent. Here we report the values at the lowest experimental temperature $T=1.5 \mathrm{~K}$.

** This is the value of the critical current at the turn over temperature $T^{*}=75 \mathrm{~K}$.

\subsection{In the "far" low critical current regime}

The possibility to have extremely low critical current density can be functional to investigate phase dynamics at extreme conditions. An example is given by a recent experiment on submicron $\mathrm{Nb} / \mathrm{AlO}_{x} / \mathrm{Nb}$ junctions [64]. Data show an anomalous $\sigma(T)$ dependence with a negative $d \sigma / d T$ over the entire temperature range. This regime can be achieved by engineering junctions with lower critical current and junction capacitance, such that the ratio $I_{c 0} / C$, which regulates $T_{\mathrm{cr}}$, is constant and the turn over temperature $T^{*}$ is lower or comparable to the quantum crossover temperature $T_{\text {cr }}$ [11]. Another example is given by Yoon et al. [65]. They have engineered $\mathrm{Al} / \mathrm{AlO}_{x} / \mathrm{Al}$ JJs in order to obtain low critical currents, of about $400 \mathrm{nA}$, and low capacitance, of about $40 \mathrm{fF}$, at the same time. In this way they have observed that TA is completely suppressed since $T^{*}$ is lower than $T_{\mathrm{cr}}$. On the other hand, by adding a shunting capacitance in the device circuit, TA regime is recovered, showing that the shunting capacitance can be used in order to tune the phase dynamics.

Junctions with intrinsically low critical current densities could represent an interesting term of comparison to study these kinds of unconventional regimes using standard micrometer junctions. Recently YBCO biepitaxial JJs have been engineered on $\left(\mathrm{La}_{0.3} \mathrm{Sr}_{0.7}\right)\left(\mathrm{Al}_{0.65} \mathrm{Ta}_{0.35}\right) \mathrm{O}_{3}$ (LSAT)

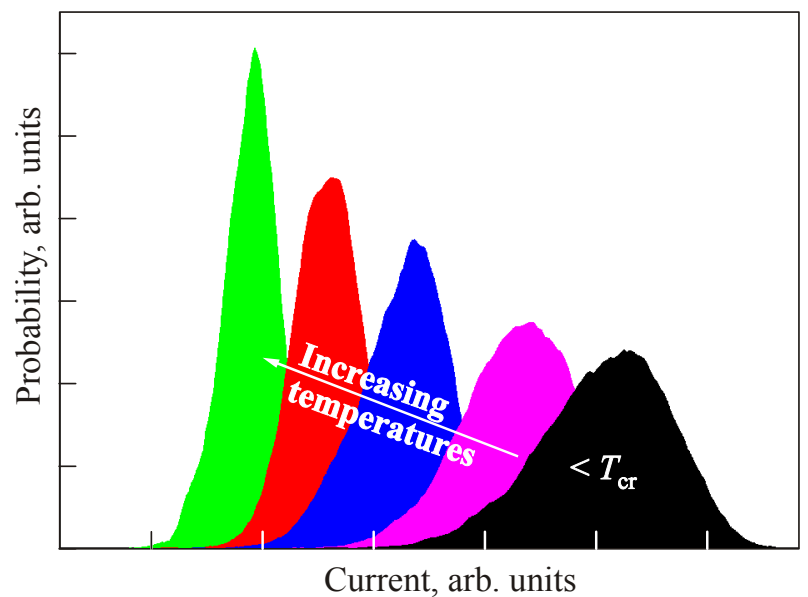

Fig. 5. Schematic representation of SCD measurements in the case of direct transition from MQT to phase diffusion regime.

substrates, responding to the needs of improving biepitaxial performances at microwaves [66]. These junctions are characterized by higher $I_{c} R_{N}$ values and by lower values of capacitance. Since LSAT substrates have a lower dielectric constant with respect to $\mathrm{SrTiO}_{3}$ substrates, this structure allows to isolate GB contribution from stray capacitance, and tends to favor moderately damped regime [66]. In a recent study [67] a direct transition from $P D$ regime to MQT has been demonstrated in these junctions. This has been made possible by selecting junctions where the crossover temperature $T_{\text {cr }}$ is higher than $T^{*}$. In these conditions thermal escape is dominated by the other contributions in their respective ranges. This behavior is easily visualized in Fig. 5 through a schematic representation of the switching current distribution as a function of temperature. Differently from the low dissipation case (see Fig. 2), the SCD broadens when lowering the $T$ and correspondingly the peak intensity decreases. Monte Carlo simulations of the thermal behavior of $\sigma$ in the PD regime could be used in order to estimate the junction quality $Q$ also in the moderately damped regime.

The whole set of data collected in these subsections is a solid framework where most of the phase dynamics can be easily classified through a study of switching current distributions. A phase diagram valid in a large range of dissipation conditions emerges as a functional guide to classify all types of behaviors and as reference for phase dynamics of novel types of junctions.

\subsection{Switching current distribution measurements in "nanostructures"}

SCD measurements have recently performed on a series of different nanostructures. Some of them are junctions and then can be easily classified in the schemes described above, and more specifically in the moderately damped regime. Some of them are simple nanowires. We believe a subtle path exists between these different systems with analogies 
and quite distinctive features. When considering that a microbridge of width of the order of the coherence length behaves as a Josephson junction [3,4], SCD measurements will turn to be more and more a direct way of discriminating the phase dynamics and the transport in nontrivial cases, which are going to be more and more common with advances in nanopatterning superconductors at extreme scales.

Supercurrent passes in graphene sheets comprised in between superconducting electrodes [68]. This is one of the nanostructured proximity-coupled Josephson systems based on conducting spacers, able in principle to be electrically tuned. Other possibilities other than graphene are offered by nanowires [70,71], carbon nanotubes [72,73] and nanocrystals [74]. In Lee et al. [68] graphene is attached to PbIn electrodes separated by a trench of $300 \mathrm{~nm}$. PbIn superconducting electrodes significantly enhances the critical current $I_{c}$ compared with commonly used $\mathrm{Al}$ (as high as $6 \mu \mathrm{A}$ in highly doped regions). The crossover from the classical to quantum regime is controlled by the gate voltage and has been found surprisingly high of the order of a few hundreds $\mathrm{mK}$. $Q$ factor is about 5-6 for all voltages. Capacitance is for instance about $35 \mathrm{fF}$ at $V=-60 \mathrm{~V}$ and seems to be not related to self-heating [75] but consistent with an effective capacitance $C_{\mathrm{eff}}=\hbar / R_{N} E_{\mathrm{Th}}$ due to diffusive motion of quasiparticles in graphene [76] $\left(E_{\mathrm{Th}}\right.$ is the Thouless energy). Phase diffusion regime has been found for all gate voltages with $T^{*}$ ranging from about $1 \mathrm{~K}(V=0)$ to $2 \mathrm{~K}$ $(V=-60 \mathrm{~V})$. Fingerprints of the Thouless energy [77] and of the minigap, commonly observed in the transport properties of nanostructures [78,79] should also somehow manifest in the switching dynamics in nanostructures.

Stochastic dynamics of superconductive-resistive switching in hysteretic current-biased superconducting nanowires undergoing phase-slip fluctuations is a topic of growing interest. Recent studies have reported phase-slip induced switching in superconducting nanowires [80-83]. In $\mathrm{Mo}_{79} \mathrm{Ge}_{21}$ nanowires of lenght ranging from $100 \mathrm{~nm}$ up to $200 \mathrm{~nm}$ [80], SDM have been used to investigate the behavior of individual quantum phase-slip events at high bias currents, observing a monotonic increase of $\sigma$ with decreasing temperature. In Al nanowires [81] of width less than $10 \mathrm{~nm}$ and length ranging from 1.5 to $10 \mu \mathrm{m}$ (with critical currents of the order of a few $\mu \mathrm{A}$ ), fluctuations in the average critical current exhibit three distinct regions of behaviors and are nonmonotonic in temperature. Saturation is present well below the critical temperature $T_{c}, \sigma$ increases as $T^{2 / 3}$ at intermediate temperatures, and a collapse is present close to $T_{c}$. The relationship between individual phase slips and switching has been also theoretically investigated [84] in order to provide a tool to study phase slips, to help establish whether they are caused by thermal fluctuations or by macroscopic quantum tunneling [85]. It has been found that although several phase-slip events are generally necessary to induce switching, there is an experimentally accessible regime of temperatures and currents for which just one single phase-slip event is sufficient to induce switching, via the local heating it causes.

\section{Conclusions}

We have focused on macroscopic quantum decay phenomena, as one of the most exciting expressions of the Josephson effect. We have in particular reviewed experiments on unconventional systems where Josephson junctions are characterized by intermediate levels of dissipation. For unconventional types of junctions, we intend both $d$-wave HTS JJs and low- $J_{c} \mathrm{NbN}$ JJs, or devices scaled to the nanosize or incorporating superconductors and nanowires or flakes as barriers. Switching current distribution measurements are a direct way of discriminating the phase dynamics and the transport also in nontrivial cases of moderate damping, which are going to be more and more common with advances in nanopatterning superconductors and in materials science with novel possibilities of synthesizing also hybrid coplanar systems. A wide vision on macroscopic quantum phenomena in a variety of complementary systems including $d$-wave junctions can promote novel arguments on the interplay of coherence and dissipation in solid state systems.

\section{Acknowledgments}

This contribution initially intended to celebrate 50 years from the discovery of the Josephson effect, also stands now as the last paper of our friend and maestro Antonio Barone. Antonio passed away before He could see the final line of the work, that however responds to the common initial planning. This manuscript is now also in his honor, touching one of his favorite topics, macroscopic quantum phenomena in Josephson junctions. This work is supported by MIUR PRIN 2009 under the project SuFET based on nanowires and HTS. We also acknowledge partial support by STREP MIDAS, Macroscopic Interference Devices for atomic and Solid State Physics: Quantum Control of Supercurrents and by a Marie Curie International Reintegration Grant No. 248933 hybMQC within the 7th European Community Framework Programme.

1. B.D. Josephson, Phys. Lett. 1, 251 (1962).

2. I.O. Kulik and K. Yanson, The Josephson Effect in Superconductive Tunneling Structures, Israel Program of Scientific Translations (Jerusalem) (1972).

3. A. Barone and G. Paternó, Physics and Applications of the Josephson Effect, John Wiley, New York (1982).

4. K.K. Likharev, Dynamics of Josephson Junctions and Circuits, New York: Gordon and Breach (1986); K.K. Likharev, Rev. Mod. Phys. 51, 101 (1979).

5. A.O. Caldeira and A.J. Leggett, Phys. Rev. Lett. 46, 211 (1981); A.J. Leggett, J. Phys. (Paris) Colloq. 39, C6-1264 (1980); A.O. Caldeira and A.J. Leggett, Ann. Phys. (N.Y) 149, 374 (1983). 
6. A.F. Andreev, Zh. Eksp. Teor. Fiz. 46, 1823 (1964) [Sov. Phys. JETP 19, 1228 (1964)]; I.O. Kulik, Sov. Phys. JETP 30, 944 (1969).

7. C.C. Tsuei and J.R. Kirtley, Rev. Mod. Phys. 72, 969 (2000).

8. T. Van Duzer and C.W. Turner, Principles of Superconductive Devices and Circuits, New York, Elsevier (1991).

9. J. Clarke and F. Wilhelm, Nature 453, 1031 (2008).

10. T.A. Fulton and L.N. Dunkleberger, Phys. Rev. B9, 4760 (1974).

11. M.H. Devoret, J.M. Martinis, and J. Clarke, Phys. Rev. Lett. 55, 1908 (1985).

12. J.M. Martinis, M.H. Devoret, and J. Clarke, Phys. Rev. B35, 4682 (1987); J.M. Martinis, M.H. Devoret, and J. Clarke, Phys. Rev. Lett. 55, 1543 (1985).

13. V.L. Ginzburg and L.D. Landau, Zh. Eksperim. i Teor. Fiz. 20, 1064 (1950).

14. H.A. Kramers, Physica (Utrecht) 7, 284 (1940).

15. R.L. Kautz and J.M. Martinis, Phys. Rev. B42, 9903 (1990); J.M. Martinis and R.L. Kautz, Phys. Rev. Lett. 63, 1507 (1989).

16. J.M. Kivioja, T.E. Nieminen, J. Claudon, O. Buisson, F.W.J. Hekking, and J.P. Pekola, Phys. Rev. Lett. 94, 247002 (2005).

17. J. Männik, S. Li, W. Qiu, W. Chen, V. Patel, S. Han, and J.E. Lukens, Phys. Rev. B71, 220509 (2005).

18. V.M. Krasnov, T. Golod, T. Bauch, and P. Delsing, Phys. Rev. B76, 224517 (2007); V.M. Krasnov, T. Bauch, S. Intiso, E. Hurfeld, T. Akazaki, H. Takayanagi, and P. Delsing, Phys. Rev. Lett. 95, 157002 (2005).

19. J.C. Fenton, and P.A. Warburton, Phys. Rev. B78, 054526 (2008).

20. Yu.M. Ivanchenko and L.A. Zilberman, Zh. Eksp. Teor. Fiz. 55, 2396 (1968) [Sov. Phys. JETP 28, 1272 (1969)].

21. A. Barone and Yu.N. Ovchinnikov, J. Low Temp. Phys. 55, 297 (1984); Yu.N. Ovchinnikov and A. Barone, J. Low Temp. Phys. 67, 323 (1987).

22. R.F. Voss and R.A. Webb, Phys. Rev. Lett. 47, 265 (1981).

23. L.D. Jackel, J.P. Gordon, E.L. Hu, R.E. Howard, L.A. Fetter, D.M. Tennant, R.W. Epworth, and J. Kurkijarvi, Phys. Rev. Lett. 47, 697 (1981).

24. W. der Boer and R. de Bruyn Ouboter, Physica B98, 185 (1980); D.W. Bol, R. van Weelderen, and R. de Bruyn Ouboter, Physica B122, 2 (1983); D.W. Bol, J.J.F. Scheffer, W. Giele, and R. de Bruyn Ouboter, Physica B113, 196 (1985).

25. R.J. Prance, A.P. Long, T.D. Clarke, A. Widom, J.E. Mutton, J. Sacco, M.W. Potts, G. Negaloudis, and F. Goodall, Nature 289, 543 (1981).

26. I.M. Dmitrenko, V.A. Khlus, G.M. Tsoi, and V.I. Shnyrkov, Fiz. Nizk. Temp. 11, 146 (1985) [Sov. J. Low Temp. Phys. 11, 77 (1985)].

27. S. Washburn, R.A. Webb, R.F. Voss, and S.M. Farris, Phys. Rev. Lett. 54, 2712 (1985).

28. D.B. Schartz, B. Sen, C.N. Archie, and J.E. Lukens, Phys. Rev. Lett. 55, 1547 (1985).

29. A. Wallraff, T. Duty, A. Lukashenko, and A.V. Ustinov, Phys. Rev. Lett. 90, 370031 (2003).
30. A.J. Leggett, in Chance and Matter: Les Houches Session XLVI, J. Souletie, J. Vannimenus, and R. Stora (eds.), Elsevier, Amsterdam (1987), p. 395.

31. Y. Nakamura, C.D. Chen, and J.S. Tsai, Phys. Rev. Lett. 79, 2328 (1997); Y. Nakamura, Y.A. Pashkin, and J.S. Tsai, Nature 398, 786 (1999).

32. J.R. Friedman, V. Patel, W. Chen, S.K. Tolpygo, and J.E. Lukens, Nature 406, 43 (2000).

33. C.H. van der Wal, A.C.J. ter Haar, F.K. Wilhelm, R.N. Schouten, C.J.P.M. Harmans, T.P. Orlando, S. Lloyd, and J.E. Mooij, Science 290, 773 (2000).

34. D. Vion, A. Aassime, A. Cottet, P. Joyez, H. Pothier, C. Urbina, D. Esteve, and M.H. Devoret, Science 296, 886 (2002); G. Ithier, E. Collin, P. Joyez, P.J. Meeson, D. Vion, D. Esteve, F. Chiarello, A. Shnirman, Y. Makhlin, J. Schriefl, and G. Schon, Phys. Rev. B72, 134519 (2005).

35. J.M. Martinis, S. Nam, J. Aumentado, and C. Urbina, Phys. Rev. Lett. 89, 117901 (2002).

36. P. Bertet, I. Chiorescu, G. Burkard, K. Semba, C.J. Harmans, D.P. DiVincenzo, and J.E. Mooij, Phys. Rev. Lett. 95, 257002 (2005).

37. M. Steffen, M. Ansmann, R. McDermott, N. Katz, R.C. Bialczak, E. Lucero, M. Neeley, E.M. Weig, A.N. Cleland, and J.M. Martinis, Phys. Rev. Lett. 97, 050502 (2006).

38. Y. Nakamura, Y.A. Pashkin, T. Yamamoto, and J.S. Tsai, Phys. Rev. Lett. 88, 047901 (2002).

39. D.A. Bennett, L. Longobardi, Vijay Patel, Wei Chen, D.V. Averin, and J.E. Lukens, Quantum Inf. Process. 8, 217 (2009).

40. L. Longobardi, Studies of Quantum Transitions of Magnetic Flux in a rf SQUID Qubit, LAP Lambert Academic Publishing (2010).

41. J. Koch, T.M. Yu, J. Gambetta, A.A. Houck, D.I. Schuster, J. Majer, A. Blais, M.H. Devoret, S.M. Girvin, and R.J. Schoelkopf, Phys. Rev. A76, 042319 (2007).

42. Y. Kubo, C. Grezes, A. Dewes, T. Umeda, J. Isoya, H. Sumiya, N. Morishita, H. Abe, S. Onoda, T. Ohshima, V. Jacques, A. Dreau, J.-F. Roch, I. Diniz, A. Auffeves, D. Vion, D. Esteve, and P. Bertet, Phys. Rev. Lett. 107, 220501 (2011).

43. M. Iansiti, A. Johnson, W.F. Smith, H. Rogalla, C.J. Lobb, and M. Tinkham, Phys. Rev. Lett. 59, 489 (1987); M. Iansiti, M. Tinkham, A.T. Johnson, W.F. Smith, and C.J. Lobb, Phys. Rev. B39, 6465 (1989).

44. F. Tafuri and J.R. Kirtley, Rep. Prog. Phys. 68, 2573 (2005).

45. T. Bauch, F. Lombardi, F. Tafuri, G. Rotoli, A. Barone, P. Delsing, and T. Cleason, Phys. Rev. Lett. 94, 087003 (2005).

46. T. Bauch, T. Lindström, F. Tafuri, G. Rotoli, P. Delsing, T. Claeson, and F. Lombardi, Science 311, 5757 (2006).

47. K. Inomata, S. Sato, K. Nakajima, A. Tanaka, Y. Takano, H.B. Wang, M. Nagao, H. Hatano, and S. Kawabata, Phys. Rev. Lett. 95, 107005 (2005).

48. X.Y. Jin, J. Lisenfeld, Y. Koval, A. Lukashenko, A.V. Ustinov, and P. Mueller, Phys. Rev. Lett. 96, 177003 (2006).

49. P.W. Anderson, Science 288, 480 (2000); Handbook of HighTemperature Superconductivity: Theory and Experiment, J.S. Brooks and J. Robert Schrieffer (eds.), Springer (2006). 
50. Y.V. Fominov, A.A. Golubov, and M.Y. Kupriyanov, JETP Lett. 77, 587 (2003); M.H.S. Amin and A.Y. Smirnov, Phys. Rev. Lett. 92, 017001 (2004); S. Kawabata, S. Kashiwaya, Y. Asano, and Y. Tanaka, Phys. Rev. B70, 132505 (2004); ibid. B72, 052506 (2005); T. Yokoyama, S. Kawabata, T. Kato, and Y. Tanaka, Phys. Rev. B76, 134501 (2007).

51. H. Hilgenkamp and J. Mannhart, Rev. Mod. Phys. 74, 485 (2002).

52. F. Tafuri, F. Miletto Granozio, F. Carillo, A. Di Chiara, K. Verbist, and G. Van Tendeloo, Phys. Rev. B59, 11523 (1999).

53. F. Lombardi, F. Tafuri, F. Ricci, F. Miletto Granozio, A. Barone, G. Testa, E. Sarnelli, J.R. Kirtley, and C.C. Tsuei, Phys. Rev. Lett. 89, 207001 (2002).

54. F. Miletto Granozio, U. Scotti di Uccio, F. Lombardi, F. Ricci, F. Bevilacqua, G. Ausanio, F. Carillo, and F. Tafuri, Phys. Rev. B67, 184506 (2003).

55. G. Rotoli, T. Bauch, T. Lindstrom, D. Stornaiuolo, F. Tafuri, and F. Lombardi, Phys. Rev. B75, 144501 (2007).

56. R. Kleiner, F. Stenmeyer, G. Kunkel, and P. Muller, Phys. Rev. Lett. 68, 2394 (1992).

57. R. Kleiner and P. Muller, Phys. Rev. B49, 1327 (1994); H.B. Wang, P.H. Wu, and T. Yamashita, Appl. Phys. Lett. 78, 4010 (2001).

58. D. Vion, M. Gotz, P. Joyez, D. Esteve, and M.H. Devoret, Phys. Rev. Lett. 77, 3435 (1996).

59. Myung-Ho Bae, M. Sahu, Hu-Jong Lee, and A. Bezryadin, Phys. Rev. B79, 104509 (2009).

60. I. Petkovic and M. Aprili, Phys. Rev. Lett. 102, 157003 (2009).

61. J. Pfeiffer, T. Gaber, D. Koelle, R. Kleiner, E. Goldobin, M. Weides, H. Kohlstedt, J. Lisenfeld, A.K. Feofanov, and A.V. Ustinov, Preprint Cond Mat. 0903.1046; J. Pfeiffer, M. Kemmler, D. Koelle, R. Kleiner, E. Goldobin, M. Weides, A.K. Feofanov, J. Lisenfeld, and A.V. Ustinov; Phys. Rev. B77, 214506 (2008).

62. L. Longobardi, D. Massarotti, G. Rotoli, D. Stornaiuolo, G. Papari, A. Kawakami, G.P. Pepe, A. Barone, and F. Tafuri, Appl. Phys. Lett. 99, 062510 (2011).

63. L. Longobardi, D. Massarotti, G. Rotoli, D. Stornaiuolo, G. Papari, A. Kawakami, G.P. Pepe, A. Barone, and F. Tafuri, Phys. Rev. B84, 184504 (2011).

64. H.F. Yu, X.B. Zhu, Z.H. Peng, Ye Tian, D.J. Cui, G.H. Chen, D.N. Zheng, X.N. Jing, Li Lu, S.P. Zhao, and Siyuan Han, arXiv:1101.2250v1 [cond-mat.supr-con] (2011).

65. Y. Yoon, S. Gasparinetti, M. Mttnen, and J.P. Pekola, J. Low. Temp. Phys. 163, 164 (2011).

66. D. Stornaiuolo, G. Papari, N. Cennamo, F. Carillo, L. Longobardi, D. Massarotti, A. Barone, and F. Tafuri, Supercond. Science and Technology 24, 045008 (2011), D. Stornaiuolo et al., unpublished (2012).

67. L. Longobardi, D. Massarotti, D. Stornaiuolo, L. Galletti, G. Rotoli, and F. Tafuri, unpublished (2012).

68. G.-H. Lee, D. Jeong, J.-H. Choi, Y.-J. Doh, and H.-J. Lee, Phys. Rev. Lett. 107, 146605 (2011); D. Jeong, J.-H. Choi, G.-H. Lee1, S. Jo, Y.-J. Doh, and H.-J. Lee, Phys. Rev. B83, 094503 (2011).
69. C. Ojeda-Aristizabal, M. Ferrier, S. Gueron, and H. Bouchiat, Phys. Rev. B79, 165436 (2009); I.V. Borzenets, U.C. Coskun, S.J. Jones, and G. Finkelstein, Phys. Rev. Lett. 107, 137005 (2011).

70. Y.J. Doh, J.A. van Dam, A.L. Roest, E.P.A.M. Bakkers, L.P. Kouwenhoven, and S. De Franceschi, Science 309, 272 (2005).

71. J. Xiang, A. Vidan, M. Tinkham, R.M. Westervelt, and C.M. Lieber, Nature Nanotech. 1, 208 (2006).

72. P. Jarillo-Herrero, J.A. van Dam, and L.P. Kouwenhoven, Nature 439, 953 (2006).

73. J.P. Cleuziou, W. Wernsdorfer, V. Bouchiat, T. Ondarcuhu, and M. Monthioux, Nature Nanotech. 1, 53 (2006).

74. G. Katsaros, P. Spathis, M. Stoffel, F. Fournel, M. Mongillo, V. Bouchiat, F. Lefloch, A. Rastelli, O.G. Schmidt, and S. De Franceschi, Nature Nanotech. 5, 458 (2010).

75. H. Courtois, M. Meschke, J.T. Peltonen, and J.P. Pekola, Phys. Rev. Lett. 101, 067002 (2008).

76. L. Angers, F. Chiodi, G. Montambaux, M. Ferrier, S. Gureron, H. Bouchiat, and J.C. Cuevas, Phys. Rev. B77, 165408 (2008).

77. J.T. Edwards and D. Thouless, J. Phys. C: Solid State Phys. 5, 807 (1972); D.J. Thouless, Phys. Rev. Lett. 39, 1167 (1977); Y. Imry, Introduction to Mesoscopic Physics, Oxford University Press (1997); C.W.J. Beenakker, Rev. Mod. Phys. 69, 731 (1997); B.L. Altshuler and P.A. Lee, Phys. Today 41, 36 (1988); R.A. Webb and S. Washburn, Phys. Today 41, 46 (1988).

78. M. Vinet, C. Chapelier, and F. Lefloch, Phys. Rev. B63, 165420 (2001); A.K. Gupta, L. Cretinon, N. Moussy, B. Pannetier, and H. Courtois, Phys. Rev. B69, 104514 (2004); H. le Sueur, P. Joyez, H. Pothier, C. Urbina, and D. Esteve, Phys. Rev. Lett. 100, 197002 (2008),

79. P. Lucignano, D. Stornaiuolo, F. Tafuri, B.L. Altshuler, and A. Tagliacozzo, Phys. Rev. Lett. 105, 147001 (2010).

80. M. Sahu, M.H. Bae, A. Rogachev, D. Pekker, T.C. Wei, N. Shah, P.M. Goldbart, and A. Bezryadin, Nature Phys. 5, 503 (2009).

81. P. Li, P.M. Wu, Y. Bomze, I.V. Borzenets, G. Finkelstein, and A.M. Chang, Phys. Rev. Lett. 107, 137004 (2011).

82. Peng Li, Ph.M. Wu, Yu. Bomze, I.V. Borzenets, G. Finkelstein, and A.M. Chang, Phys. Rev. B84, 184508 (2011).

83. M. Tinkham, J.U. Free, C.N. Lau, and N. Markovic, Phys. Rev. B68, 134515 (2003).

84. N. Shah, D. Pekker, and P.M. Goldbart, Phys. Rev. Lett. 101, 207001 (2008).

85. W.A. Little, Phys. Rev. 156, 396 (1967); J.S. Langer and V. Ambegaokar, Phys. Rev. 164, 498 (1967); D.E. McCumber and B.I. Halperin, Phys. Rev. B1, 1054 (1970); N. Giordano, Phys. Rev. Lett. 61, 2137 (1988); A. Bezryadin, C.N. Lau, and M. Tinkham, Nature 404, 971 (2000); C.N. Lau, N. Markovic, M. Bockrath, A. Bezryadin, and M. Tinkham, Phys. Rev. Lett. 87, 217003 (2001); A. Rogachev, A.T. Bollinger, and A. Bezryadin, Phys. Rev. Lett. 94, 017004 (2005); F. Altomare, A.M. Chang, M.R. Melloch, Y. Hong, and C.W. Tu, Phys. Rev. Lett. 97, 017001 (2006). 